\title{
À propos des révoltes et révolutions de la fin du
} XVIIIe siècle

Essai d'un bilan historiographique

Guy Lemarchand

\section{(2) OpenEdition}

\section{Journals}

Édition électronique

URL : https://journals.openedition.org/ahrf/2236

DOI : $10.4000 /$ ahrf.2236

ISSN : 1952-403X

Éditeur :

Armand Colin, Société des études robespierristes

Édition imprimée

Date de publication : 1 juin 2005

Pagination : 145-174

ISSN : 0003-4436

Référence électronique

Guy Lemarchand, "À propos des révoltes et révolutions de la fin du XVIIle siècle », Annales historiques de la Révolution française [En ligne], 340 | avril-juin 2005, mis en ligne le 27 avril 2006, consulté le 22 avril 2022. URL : http://journals.openedition.org/ahrf/2236 ; DOI : https://doi.org/10.4000/ahrf.2236

Ce document a été généré automatiquement le 22 avril 2022

Tous droits réservés 


\title{
À propos des révoltes et révolutions de la fin du XVIIIe siècle
}

\author{
Essai d'un bilan historiographique
}

\section{Guy Lemarchand}

1 La question mise au programme des concours du C.A.P.E.S. et de l'agrégation d'histoire pour 2005-2006 recoupe le champ chronologique des Annales historiques de la Révolution française. Intitulée "Révoltes et Révolutions en Europe (Russie incluse) et aux Amériques 1773-1802 ", elle a suscité en quelques mois pas moins de dix publications ${ }^{1}$ destinées à couvrir en large partie le sujet, sept étant collectives et trois individuelles, soit au total à peu près 3000 pages imprimées dont, sans les tables des matières, bibliographies et documents, plus de 2500 pages de texte. Se sont ainsi exprimés 56 historiens, certains donnant jusqu'à trois contributions, la majorité en fournissant une seule. Évidemment pour construire son étude, chacun a d'abord puisé dans ses recherches personnelles, mais la plupart ont eu le souci de rédiger des mises au point qui tiennent compte d'autres travaux anciens et récents sur le thème traité, et fréquemment de confronter les points de vue. C'est dire que, en dépit de scories dues à la précipitation de l'élaboration et de la correction des épreuves d'imprimerie pour lesquelles les auteurs ont disposé d'à peine six mois, la lecture de ces ouvrages apporte d'abord un bilan des connaissances sur une matière vaste par son assise géographique et qui, si elle est restreinte à trois décennies, se situe dans une période de bouleversements rapides et intenses du monde. De plus, le nombre et la variété des méthodes et des types d'explication mises en œuvre par les intervenants permet, dans une certaine mesure, d'appréhender des débats de fond en cours et les différences d'orientation dans les interprétations, c'est-à-dire de saisir les courants historiographiques actuellement aux prises. Par conséquent, la grande majorité des contributions dans ces volumes dépasse le but pratique immédiat de préparation aux concours et offre des synthèses qui peuvent donner réflexion aux chercheurs.

I - Le cadre de la problématique d'ensemble

2 Au-delà des initiatives commerciales des éditeurs, on peut se demander pourquoi la question a mobilisé autant les collègues. C'est, me semble-t-il, qu'elle présentait dans le 
paysage historiographique d'aujourd'hui un caractère relativement novateur et hardi. En effet elle oblige à adopter une démarche globalisante qui était en faveur dans les années 1960, mais qui, depuis 1980, a été soumise à une critique souvent vive si ce n'est justifiée par une partie des historiens et par nombre des médias. On la jugea alors artificielle, les catégories de la méthode quantitative qui en étaient partie intégrante n'étant que des créations intellectuelles plaquées sur une réalité foisonnante, elle était donc réductrice par rapport à la complexité humaine et même archaïque par rapport aux progrès de la psychosociologie et de l'anthropologie, pour ne pas dire en plus totalitaire dans sa visée. Cependant, s'il n'était certainement pas dans l'esprit des jurys de concours de faire dresser un corpus érudit aussi complet que possible de toutes les émeutes et révoltes de la période, le seul rapprochement des deux termes révolte et révolution mis au pluriel suggérait de ne pas s'en tenir aux faits événementiels à caractère le plus souvent immédiatement politique. Il était nécessaire d'envisager les conditionnements divers des faits, conjoncturels et structurels, non seulement culturels mais également économiques, démographiques, sociaux, voire écologiques. D'autre part, l'étendue du cadre géographique retenu avec la mention des États européens colonisateurs et de l'Amérique coloniale suppose de ne pas se contenter de juxtaposer les études par pays les uns après les autres, mais de pratiquer ce qui est encore peu répandu dans l'école historique française, quoiqu'en développement récent: une histoire comparée internationale à laquelle répugnait le positivisme d'autrefois. Pour cela un minimum de théorisation s'avère quasi indispensable, en définissant les concepts de base - ne serait-ce qu'autour des mots émeute, sédition, révolte, révolution - et en dessinant des schèmes explicatifs, ce que l'antisociologisme de la pensée libérale dominante actuelle n'aime pas. De même, contre certains tenants d'une histoire culturelle privilégiant subjectivité et instant, l'étude d'événements à composantes multiples comme les grandes révoltes paysannes ou les journées révolutionnaires urbaines nécessite de considérer à la fois la courte durée et la longue, et, au-delà des représentations, d'aller aux objets représentés eux-mêmes dans leur chair matérielle et sociale.

31773 donne d'emblée une dimension presque mondiale à la question : la « tea-party » de Boston et la révolte de Pougatchev. 1802 correspond à la paix d'Amiens et la fin provisoire des croisades des monarchies contre la France ainsi que la consolidation du pouvoir dans l'hexagone avec le Consulat à vie, ce qui souligne le rôle majeur de la Révolution française dans l'histoire de la période. Toutefois l'analyse de la violence dans ces trente années ne peut se satisfaire totalement d'une durée aussi brève. En fait, enserrant les deux révolutions principales de la fin des Temps Modernes, ces limites à la fois disent l'essentiel et bornent le travail des étudiants. Elles n'empêchent pas les auteurs des ouvrages répertoriés ici de remonter éventuellement bien plus haut. Comment traiter de l'indépendance américaine sans saisir l'accumulation d'incidents créant peu à peu une rupture avec la Grande-Bretagne à partir de la fin de la guerre de Sept Ans ou même de la guerre de Succession d'Autriche avec la politique de lord Halifax, et même pour quelques traits de mentalité des colons en rébellion, on pourrait aller jusqu'au débarquement du Mayflower. Parfois également il est utile de franchir la barrière de 1802, par exemple afin de saisir les modalités et étapes de la formation du sentiment national unitaire italien jusqu'au Risorgimento, ce qui permet de mieux caractériser l'originalité du moment de la première occupation française de 1796-1799.

4 Évidemment, aucun de ces livres ne vise à couvrir exactement tous les aspects de la question. Néanmoins, en ce qui concerne l'extension géographique, la dure contrainte 
de sortir pour l'étude de l'Europe du franco-centrisme, c'est-à-dire une Europe limitée à quelques États voisins de notre pays, a été respectée plus ou moins, mais certaines régions demeurent encore dans l'ombre. Puisque vers l'Est la question étend son champ jusqu'à l'Oural, comme d'ailleurs le précise la bibliographie - quoique insuffisante semi-officielle parue dans Historiens et géographes en juillet 2004, on est obligé de se pencher sur les travaux des pays ex-communistes réalisés avant 1990 par des historiens qui étaient loin d'être dans leur très grande majorité ni des sbires staliniens ni des perroquets brejneviens et qui publiaient des synthèses de leurs recherches dans des ouvrages périodiques, tels ceux rédigés à l'occasion des Congrès Internationaux des Sciences historiques, et des revues comme les Acta Poloniae Historica, Études Balkaniques (Sofia) ou la Revue Roumaine d'Histoire, dans des langues aussi appréhensibles en France que le français ou l'anglais. Or il faut bien dire que l'on trouve très peu de mentions de ces livres et articles dans les pages ici recensées. La Scandinavie du despotisme éclairé de Struensee, Bernstorff et Gustave III et des grandes réformes agraires d'enclosures n'est guère abordée, le Danemark n'étant même pas mentionné. À l'autre bout du vieux continent, une terre par excellence de soulèvements paysans et de révoltes de gouverneurs contre le pouvoir central, la Roumélie ottomane, est passée sous silence, ainsi que les Principautés roumaines vassales de la Porte. Pourtant la figure des haïdouks ou le personnage d'Ali de Tepelen popularisé par A. Dumas, et les réformes agraires de Constantin Mavrocordat quoiqu'antérieures à 1773, font bien partie du sujet. Seul R. Calvet (Calvet) indique la naissance de la littérature nationale grecque avec Katartzis ou Koraes. Mais est absente la quatrième révolution de la période à côté de l'américaine, la française et l'haïtienne, le soulèvement serbe de février 1804 qui va l'emporter dès 1815 et qui se prépare dès la fin de la guerre russo-turque de 1787-1792. Quant au Nouveau monde, qui va alors de Québec à Buenos-Aires, le point de vue adopté reste majoritairement européo-centriste dans la mesure où, en dehors des Antilles françaises esclavagistes, ce qui retient l'attention est le plus souvent l'Amérique des colons. Sans doute y-a-t-il ici une difficulté pour l'historien à utiliser des travaux qui sont surtout le fait de l'ethnologie et de l'anthropologie. En tout cas, on constate que l'étude des révoltes indiennes est limitée souvent aux grands soulèvements du Pérou et Bolivie des années 1780. Pour l'Amérique espagnole, les troubles du Mexique, en particulier lors des crises de subsistances, n'apparaissent pas, pas davantage que les révoltes des marges de l'empire hispanique avec la résistance à la pénétration coloniale des missions franciscaines du Nouveau-Mexique, du Texas et du Colorado et les rapts d'indigènes pour le travail forcé sur les terres nouvellement conquises, auxquels répliquent les raids d'Apaches et Comanches brûlant les villages jusqu'aux années 1785-1790. Pourtant plus connus, les Araucans du sud du Chili sont également absents. D'un abord bibliographique plus difficile, il est vrai, ne sont guère mentionnés non plus les Indiens du Brésil malgré, entre autres, l'agitation autour de la dissolution des réserves des Jésuites. Par contre, à cause $\mathrm{du}$ poids de Saint-Domingue et de sa révolution, l'esclavage des Noirs est longuement étudié, avec cependant peu d'intérêt porté à la rivale britannique de la colonie française, la Jamaïque.

Du point de vue du caractère global des analyses, qui nous rapproche de la volonté d'histoire totale des Annales de M. Bloch et L. Febvre, l'accent est mis, comme le libellé de la question y invite, sur le politique et le culturel. Mais les racines matérielles et humaines des crises et agitations ne sont généralement pas omises, même si dans les explications des faits certains accordent beaucoup plus d'importance à l'enchaînement 
des idées et des systèmes politiques qu'aux conditionnements sociaux. Ainsi se marque un recul d'une approche purement idéique fort développée dans les années 1980. Pour autant, l'utilisation du concept d'idéologie demeure floue et le recours aux instruments intellectuels qu'on peut tirer de l'œuvre de P. Bourdieu auquel on ne peut pas ne pas songer ici, reste rare, ce qui indique les limites de l'effort théorique tenté. On se souvient d'ailleurs qu'un essai d'inventaire des emplois possibles des travaux du sociologue par les historiens, mené courageusement par la Société d'Histoire Moderne et Contemporaine (2), avait tourné court. De plus, si dans les développements l'économique trouve à peu près une place, il n'apparaît fréquemment que de façon très rapide et les vieux graphiques évocateurs du mouvement des prix des grains et des salaires ou bien ceux sur le ciseau des prix de l'économie de plantation générateur de la crise qui se dessine pour les colons des Antilles comme du Mexique, ne sont pas repris. Plus nette est la quasi disparition de la monnaie et du crédit. Sans doute la question du concours implique de ne pas se livrer à un récit chronologique complet des deux révolutions principales. Toutefois, il est notable qu'à propos des États-Unis de la période confédérale une partie de la tension intérieure, des révoltes et de l'instabilité politique provient de l'inflation : l'émission accélérée de billets en dollars par le gouvernement central, puis dans certains États, pèse sur les échanges et devient un enjeu social. Alors que le numéraire se cache, que l'étranger refuse de consentir de nouveaux prêts et que la production civile tarde à redémarrer faute de débouchés, faut-il relancer la consommation en augmentant la masse monétaire et soumettre à des moratoires les dettes des particuliers appauvris par la guerre ou faut-il au contraire créer des impôts pour assainir les finances de manière à stabiliser la monnaie et rassurer les détenteurs de capitaux? La révolte de Shays de 1786 est toujours citée, mais comment la comprendre sans examiner ce problème ? Pour la Révolution française, chacun connaît l'importance de la question de l'assignat et de l'échec du mandat territorial. Pourtant, sauf S. Bianchi (Bianchi), il n'y est que rarement fait allusion et le plus souvent de façon sommaire. Le livre de F. Crouzet (3), essentiel sur la question, n'est pas toujours porté dans les bibliographies, alors que, quelques réserves que l'on puisse avoir sur ses jugements généraux, il donne une synthèse solide et facilement lisible. Seuls J.-P. Poussou (Poussou 1) et R. Calvet (Calvet) évoquent, mais très brièvement, la discussion des années du Bicentenaire sur les conséquences économiques de la Révolution, quoique cela fasse tout de même partie de la question. N'y a-t-il pas ici un effet de cette tendance, qui s'est instaurée depuis 1980, à faire de l'histoire économique, à la manière anglo-saxonne, un domaine à part du reste de la société, comme on le faisait dans l'entre-deux-guerres, une curiosité de haute technicité (!) qu'on laisserait à quelques spécialistes patentés, ce qui représente une mutilation de la réflexion historique. Car il semble bien que les hommes, en même temps qu'ils pensent, inventent constitutions et systèmes philosophiques ou se soulèvent contre les autorités, n'en continuent pas moins à manger et se vêtir tous les jours. Le « tout culturel » n'est peut-être pas tout. Certains observateurs verront peut-être dans une telle orientation au moins un signe d'indépendance vis-à-vis du vent ambiant monétariste. N'est-ce pas plutôt une conséquence de la pénétration du néo-libéralisme qui reconstruit un «homo economicus » isolé de la réalité sociologique et fractionne le tout social en instances parfaitement autonomes les unes par rapport aux autres?

6 Enfin une véritable lacune est, ce qui aurait été impensable il y a trente ans, l'absence fréquente de considération du facteur démographique en Europe, particulièrement en Italie et en Pologne, ou dans les treize colonies anglaises d'Amérique, ou à propos de la 
reprise du peuplement indien au XVIIIe siècle comme facteur de tension dans l'empire espagnol. Dans ce dernier cas, une cause de développement des émeutes et révoltes, aussi bien au Pérou qu'au Mexique, est le grignotage des terres communes indiennes ( ${ }^{4}$ ), alors que celles-ci apparaissent déjà trop restreintes du fait de l'augmentation de la population pendant la seconde moitié du XVIIIe siècle. Cette attaque des colons est ellemême provoquée par leur propre accroissement démographique, plus modéré que celui des Indiens, mais réel, et par l'expansion de l'économie marchande qui pousse à la mise en valeur de terres nouvelles, en particulier pour l'exportation.

7 Par contre, signe des temps encore, mais dans le sens positif cette fois, les femmes en tant que telles et sans aller jusqu'à une " gender history " discutable, ont droit à des exposés systématiques, pour conclure d'ailleurs à une amélioration de leur statut et de leur sort matériel pendant la période, mais faible et inégale suivant les pays.

II - De l'émeute à la révolution

8 Un accord général parmi les auteurs se fait pour définir l'émeute comme un acte ponctuel de quelques jours sur un espace de localités peu nombreuses, tandis que la révolte mobilise des foules de plusieurs milliers de manifestants, dure plus longtemps pour couvrir une ou plusieurs provinces et se donne pour objet de modifier une fois pour toute une situation jugée injuste. Y.-M. Bercé (Poussou 2) note qu'elle suppose un minimum d'organisation avec des chefs, même improvisés. La distinction entre troubles ruraux et troubles urbains s'avère ne pas être seulement une commodité rhétorique pour la clarté de l'exposé. Elle correspond à une spécificité de chaque catégorie. Les motivations des premiers sont plutôt liées à la contestation antiseigneuriale et aux oppositions religieuses, leurs objectifs sont généralement à assez court terme. Les seconds sont dictés surtout par le problème des subsistances, les approvisionnements en ville étant plus difficiles qu'à la campagne, et par la politisation, la cité étant par excellence lieu de rencontre et de discussion. Néanmoins, il ne convient sans doute pas de pousser trop loin une telle distinction car, même dans l'Ancien Régime, les contacts entre plat-pays et villes sont nombreux: nombre de ruraux viennent fréquemment dans la cité au marché, ou à la recherche d'emplois ou de secours temporaires, ou pour des rapports avec une administration. En sens contraire, l'encadrement de la proto-industrie suppose autant des déplacements de citadins vers les villages que l'inverse, et, surtout, en particulier lors des crises de subsistances, on voit des habitants des villes battre la campagne à la recherche de stocks de denrées.

9 Le retentissement de la publication récente de la somme de J. Nicolas sur les troubles en France aux XVIIe-XVIIIe siècles ${ }^{5}$ contribue à faire prendre en considération l'émeute à côté de la révolte et à s'interroger pour savoir si des faits de petite taille peuvent avoir des effets durables et importants tels que obliger un gouvernement à changer de politique sur la question soulevée par les manifestants. Comme R. Calvet qui s'appuie même sur Marx et $\mathrm{A}$. Touraine (Calvet), chacun convient que le conflit est inhérent à la vie interne de toute société et qu'il ne débouche pas nécessairement sur la révolte et encore moins sur la révolution. La France, rappelle-t-on, a connu de grandes révoltes au XVIIe siècle sans renverser la monarchie absolutiste, elles s'éteignent sous Louis XIV et sont remplacées tout au long du XVIIIe siècle par une poussière de troubles divers plus réduits. Est-ce à dire que l'accroissement du nombre des émeutes comme encore en France à partir des années 1760 et, semble-t-il, en Grande-Bretagne, soit sans signification sur l'état des formations sociales, sur le plan tant économique que socio- 
politique, et qu'il ne puisse pas y avoir à la longue de répercussion sur la ligne suivie par les pouvoirs ainsi que le suggèrent Y.-M. Bercé et J.-P. Poussou (Poussou 2) ? Certes une vague d'incidents même assez étendue comme la « Guerre des farines » de 1775 n'a pas conduit à la remise en cause immédiate de la liberté du commerce des grains proclamée par Turgot en 1774. Mais tel n'est pas toujours le cas. R. Calvet remarque qu'en Suède - pays moins agité que la France - après les émeutes de subsistances de 1799-1800, les expériences de remembrement destinées à augmenter la production agricole et menées par de grands propriétaires agronomes paraissent se trouver justifiées en dépit des dégâts sociaux qu'elles entraînent, et sont adoptées en 1803-1807 des lois d'enclosure qui accélèrent ces opérations. On ne peut pas ne pas noter non plus que la décision de convocation des États généraux, puis celle du doublement du tiers état, en dépit de l'opposition du roi et de la Cour, sont prises, tous les auteurs le constatent, dans une atmosphère marquée par des incidents multiples de 1787 au printemps 1789 où se heurtent les foules et les forces représentant l'autorité royale, ou bien comme à Rennes, où se heurtent fils de bourgeois et jeunes aristocrates. Ces troubles sont-ils pour rien dans l'infléchissement de l'attitude du pouvoir?

Une conséquence des émeutes et surtout des révoltes est parfois de pousser ce qu'on a appelé la "révolution d'en haut ", c'est-à-dire les expériences de despotisme éclairé. Certains historiens y ont vu le moyen d'éviter les « révolutions d'en bas ». Grâce à leur énergie et leur art du gouvernement, des monarchies auraient su mener une politique avisée de réformes et de modernisation des structures de leur pays, en contenant l'impatience désordonnée, obscurantiste et meurtrière des foules populaires qui ont sévi pendant la Révolution française ou, plus tard, dans la révolution russe. En fait, lorsqu'on examine de près la chronologie des règnes des souverains éclairés, on s'aperçoit que nombre des mesures qu'ils ont prises ont été adoptées après des émeutes et des révoltes, ou au moins dans un climat d'agitation populaire. Même si certains rois et ministres sont motivés par leur adhésion aux Lumières, beaucoup de leurs actes sont dictés par l'opportunité. M. Biard (Biard) note que leur réforme la plus commune, la libération du commerce des grains, relève bien de l'influence de la physiocratie et du libéralisme anglo-saxon, mais souvent elle se retourne contre eux en déclenchant des troubles en protestation devant le développement des stockages et transports spéculatifs, ce qui peut entraîner la suspension de la décision. Pour apprécier l'étendue et la durabilité des réformes de ces souverains, il faut souvent franchir largement la barrière de 1802. Au Portugal, il ne reste presque plus rien de la politique de Pombal sous Maria I dans les années 1780. En Espagne, Charles III, en dépit d'une action limitée sur les communaux et un contrôle accru sur les évêques, n'a touché ni au déséquilibre entre les régions, ni à la toute puissance de l'Église et de la haute noblesse, sources essentielles de blocage. Et après Joseph Bonaparte et la séquence des Cortes de Cadix en 1812, Ferdinand VII peut déployer une réaction brutale. Il en est de même dans le royaume de Naples, où la facilité de la conquête française de 1799 a montré la fragilité des nouveautés introduites par Ferdinand IV, qui sont d'ailleurs suivies dès 1799 du retournement de sa politique. En Russie, si Catherine II continue les réformes après la révolte de Pougatchev, celles-ci prennent un tour de plus en plus conservateur et autoritaire, tandis que l'action d'Alexandre II apparaît plus velléitaire que réelle, ce qui mène à la crise décabriste de 1825. En Prusse, Frédéric II laisse en héritage en 1786 une lourde bureaucratie, un prélèvement accablant sur les ressources du pays au profit de l'armée, et même un appauvrissement qui excite un banditisme rural. À terme, ce sera l'écroulement de 1806. Dans les États des Habsbourg, c'est l'annulation de ses décrets 
par le plus convaincu des rois philosophes et qui est allé le plus loin, Joseph II, à son lit de mort en février 1790, devant le développement des désordres en Hongrie, en Bohème et dans les Pays-Bas. Même en Scandinavie où - et là seulement - a été mise en place une réforme agraire lente, mais réelle, en faveur des enclosures, qui d'ailleurs comporte des dégâts sociaux, il y a revirement en Suède après la régence du duc Charles (1792-1796) relativement ouverte au vent de la Révolution française, avec Gustave IV Adolphe qui aboutit au soulèvement de 1809 ; au Danemark, l'épisode libéral et créateur commencé en 1786 avec la régence du prince Frédéric, s'arrête à partir de 1799 et plus nettement encore avec la guerre de 1807-1809. Seule des grands États, sans despotisme éclairé et sans révolution, la Grande-Bretagne connaît des troubles classiques assez violents de 1770 à 1789, mais ensuite c'est une tension que P. Dupuy (AHM) qualifie de subversive qui se développe, s'attaquant au régime, avec un maximum dans les années 1795-1802. Pitt n'écrase le mouvement qu'en misant non seulement sur la répression policière et judiciaire, mais en favorisant un contremouvement populiste conservateur "King and Church », d'autant plus virulent que le danger de débarquement français est réel. La situation reste finalement bloquée jusqu'aux années 1830 et la réforme électorale de 1832. Par conséquent, le constat de l'échec de l'État autoritaire comme de l'État libéral à la fin du XVIIIe siècle et au début du XIXe siècle est général, et après 1791 la réaction des Anti-Lumières l'emporte à cause de la crainte de la contamination venue de France.

11 Il faut se garder de toute téléologie, comme le relève J.-P. Poussou (Poussou 2), et admettre qu'il n'y a évidemment aucun automatisme dans la relation émeute-révolte, pas davantage que dans celle émeute-révolution ou révolte-révolution. La question posée par S. Bianchi (Bianchi) - pourquoi la révolte de Pougatchev et la "Grande Peur » française de 1789 ont-elles des résultats aussi contradictoires ? - indique la méthode à suivre : l'explication par la seule "promptitude à l'échauffement» (terme venu du XVIIe siècle) des foules est recourir à un invariant insuffisant, plus verbal que réellement analytique, et il faut se référer aux contextes dans leur totalité. D'un côté, un régime tsariste autoritaire puissant et vainqueur, des minorités ethniques rejetées par les russes et une seigneurie qui écrase la paysannerie, de l'autre, en France, une monarchie en crise morale dont l'armée se délite, comme le précise 0 . Chaline (Poussou 2), une bourgeoisie de plus en plus nombreuse et critique, et des paysans libres revendiquant l'abolition de fait de charges privées à l'inutilité évidente. En réalité partout, à considérer la succession des années, les choses paraissent de prime abord ne pas changer. Ainsi, suivant D. Pétré-Grenouilleau (Poussou 2), le marronnage sévit largement aux Antilles ou à Surinam bien avant 1773, et il ne met pas en cause l'esclavage dans son principe. Colons et gouverneurs finissent même parfois par s'en accommoder et passent des accords de coexistence avec des rassemblements rebelles. Néanmoins, avec le développement de la traite, l'expansion de ces sortes de maquis et les raids qu'ils pratiquent contre les plantations, avec le déséquilibre croissant entre les effectifs des Blancs et ceux des populations dépendantes contribuent à la montée de la peur des Européens, sentiment qui renforce après 1788 leur propension à l'intransigeance et à la cruauté en face de ceux qu'ils ont toujours vus comme de simples outils. Le mouvement démographique encore fournit donc une des clés d'explication des comportements.

12 Il en va de même d'une autre composante de ce contexte : le facteur économique. Même s'il n'est pas toujours aussi présent qu'il serait probablement souhaitable dans ces livres, les auteurs relèvent son action dans de nombreux épisodes, même là où la 
revendication principale des mutins n'est pas matérielle ou fiscale. À propos du soulèvement des patriotes des Provinces-Unies contre le régime du stathouder Guillaume V à la suite de la désastreuse guerre avec l'Angleterre (1780-1784) et des incidents violents les opposant aux Orangistes, il y a entre autres, pour F. Antoine (Bourdin - Bianchi), la paupérisation liée au chômage provoqué par la perte de débouchés extérieurs, ce qui entretient l'effervescence. Par ailleurs, il est remarquable que parmi les huit auteurs qui traitent ou évoquent l'Indépendance américaine, tous développent longuement l'origine économique des heurts de plus en plus violents entre les colons et le gouvernement britannique, depuis le Sugar Act de 1764 jusqu'aux Intolerable Acts de 1774 fermant le port de Boston, et ils ne mentionnent pas la thèse des historiens « révisionnistes » américains des années 1960, longtemps très en faveur aux États-Unis, D.J. Boorstin, E. Morgan, R.P. Thomas ou B. Baylin, qui ont minimisé systématiquement les contradictions d'intérêts entre la nouvelle politique impériale de Londres et ses sujets du Nouveau monde, au profit des oppositions de conceptions politique et morales entre les deux parties, ce qui était plus flatteur pour les patriotes et pour la renommée des États-Unis au XXe siècle.

13 Pour autant, il n'est question pour personne de nier le rôle du facteur idéologique dans le déclenchement de nombre de troubles de cette fin du XVIIIe siècle. Ce qui implique d'abord, comme nos livres n'y manquent pas, de revisiter la notion de «révolution atlantique » ou " occidentale » lancée en 1955 par J. Godechot et R. Palmer, reprise de façon plus nuancée par le premier dans la Grande nation (1956). On ne peut en effet qu'être frappé par la succession des secousses de part et d'autres de l'Océan atlantique qui se produisent à partir de la première crise de la République de Genève en 1766 jusqu'à la Révolution française et la révolution brabançonne et même jusqu'à l'Indépendance de l'Amérique latine dans les années 1810, tous mouvements qui, ainsi que le souligne M. Dorigny (Dorigny), ont un point commun très net: il s'agit de soulèvements populaires au nom de la liberté et même du droit naturel. Évidemment, située dans le début de cette séquence d'une cinquantaine d'années, intéressant un pays de plus de 2 millions d'habitants, gros fournisseur de denrées et qui réussit à vaincre une des plus grandes puissances d'Europe, la révolution américaine encourage ceux qui sur le Vieux continent se sentent opprimés, et elle leur fournit mots d'ordre et slogans revendicatifs dont on suit aujourd'hui le cheminement. Il apparaît que sur ce plan - et seulement sur celui-là - il y a plutôt complémentarité qu'opposition entre les deux principales révolutions de la période. La seconde, la française, marque toutefois, selon l'expression de J. Bernet (Bourdin - Bianchi), une formidable accélération du mouvement en Europe et dans les Caraïbes, en même temps qu'elle pousse beaucoup plus loin l'élaboration des principes d'universalisme et de démocratie. Il est vrai que l'exemple américain a inspiré à des philosophes français avant 1789 des livres qui en tracent un tableau positif, Mably en 1784 ou Condorcet en 1787, ou que les patriotes hollandais citent fréquemment les États-Unis pour réclamer de faire cesser la vassalité de fait des Provinces-Unies vis-à-vis de l'Angleterre, ou encore que la Déclaration d'indépendance des États Belgique de 1790 reprend des termes de la Déclaration d'indépendance américaine. On est donc amené à porter une attention particulière à des objets que l'histoire culturelle a récemment mis au premier plan, les supports matériels et sociaux de la diffusion de la pensée et de la "République des lettres ", presse internationale, en particulier de langue française - secteur par excellence des Lumières - recensée par É. Wauters (Biard), caricatures, récits de voyage aux Amériques et traités d'arithmétique politique, sociétés de lectures, académies, bibliothèques, 
salons, loges maçonniques, clubs et réseaux. Ne faut-il pas ajouter à cette liste ce qui n'est pas toujours évoqué, le commerce à grande distance et les voyages d'affaires à l'occasion desquels les hommes peuvent échanger des idées, c'est un truisme grossier, à côté des marchandises ? Dans le sillage de J. Godechot, on suit la formation des groupes de réfugiés politiques chassés de chez eux après leur défaite, leurs pressions sur le gouvernement français et leur propagande en direction de leur pays, en attendant de les retrouver à la tête des républiques-sœurs. Cependant il apparaît aujourd'hui que l'influence des États-Unis sur la Révolution française a été restreinte et que les anciens soldats de Rochambeau et La Fayette sont loin d'avoir été tous des amis fervents de la liberté et des partisans de la Révolution en France. D’ailleurs, la détérioration des relations diplomatiques entre Paris et le gouvernement fédéral après 1790 n'a sans doute pas été favorable. En fait les mouvements dans chaque pays répondent d'abord à des situations locales spécifiques, et s'il existe quelques convergences entre eux, il y a également beaucoup de différences.

Du même coup, les patriotes étrangers dénommés trop commodément " jacobins » ne sont pas la copie conforme des Jacobins de Paris. Le terme d'ailleurs a été et demeure encore dévoyé, Ch. Peyrard compte 6900 occurrences sur Internet (AHM). Il a été employé à partir de 1791 d'abord par les adversaires du club, avec une connotation péjorative. Il y a eu effectivement contacts et échanges entre les uns et les autres, mais chacun, dans la mesure où il a un projet, ne reprend dans l'expérience française que ce qui lui semble adaptable à son pays. Et les États satellites de la France, s'ils se dotent en premier lieu d'une constitution inspirée par celle de l'an III, n'en ont pas moins leur vie propre. Selon le thème de la "révolution passive » lancé par les historiens libéraux italiens, dans les républiques italiennes il n'y aurait eu aucune adhésion, hors d'une très petite minorité, aux idéaux nouveaux et on n'aurait qu'importé et imposé sous la férule française les lois et institutions de la Grande Nation. On pourrait transposer l'idée à la Suisse, l'Allemagne, voire la République Batave ou les départements belges. Cependant, depuis vingt ans cette vue est apparue schématique, simplifiant à l'excès la diversité et la complexité des situations. En fait, dès avant l'arrivée des troupes françaises en 1792 ou en 1796-1799, il y a eu déjà dans ces pays des émeutes, voire des révoltes qui montraient à la fois un mécontentement populaire, une attente favorable des nouvelles de la Révolution à Paris et un début d'organisation patriotique autochtone. Et pendant ce demi-siècle les révoltes et les révolutions à leur suite se sont d'autant plus étendues que les pouvoirs en place, les monarchies autoritaires encore liées à des structures sociales et idéologiques féodales et le régime britannique constitutionnel sont restés bloqués dans les principes anciens, voulant dans les années 1770 maintenir l'équilibre entre les princes aux dépens du droit des peuples et prêts à écraser toute velléité de changer le statu quo politique et social. Dans l'esprit des gouvernements français et espagnol l'aide fournie aux Insurgents américains était un coup porté à la puissance britannique et non un geste en faveur de la liberté. De même la Prusse écrase en 1787, avec l'accord de Georges III, la révolution des Provinces-Unies, ou l'Autriche réprime en 1790 le soulèvement dans l'évêché de Bale.

De toute façon, remise à l'honneur depuis une vingtaine d'année, une bonne vieille méthode apparait ici particulièrement fructueuse : M. Dorigny (Dorigny) relève l'utilité de la biographie pour apprécier la mobilité géographique des idées et les transferts culturels d'un pays à l'autre, avec par exemple le personnage de Miranda, du Vénézuela aux États-Unis puis en France et à nouveau à Caracas. Il en va de même d'ailleurs pour saisir à l'intérieur d'une même nation l'évolution rapide de la situation à travers la 
carrière et les transformations d'individus occupant successivement des places importantes, comme le note J.-P. Poussou (Poussou 1) pour la France de la fin du XVIIIe siècle. Ainsi que l'a signalé T. Tackett à propos des Constituants: on ne naît pas révolutionnaire, on le devient dans un certain environnement ${ }^{6}$.

III - Le phénomène révolutionnaire

La révolution dépasse évidemment la révolte: parce que plus longue dans le temps, embrassant tout un État ou au moins une partie étendue, elle met à bas l'ordre sociopolitique pour en établir un autre. Ses réformes sont donc rapides, profondes et - trait sur lequel les auteurs insistent particulièrement - irréversibles. C'est la subversion provoquée par l'irruption de la masse de la population, en particulier les couches populaires, qui aboutit à un tel résultat. Cette intervention pose la question de la violence qui, dans la tradition des pamphlétaires et historiens conservateurs, serait le propre du phénomène révolutionnaire. À cet égard, un thème récurrent repris par exemple par G. Gusdorf ${ }^{7}$ et qui affleure dans les dix livres, exonère la révolution américaine du reproche d'avoir versé le sang, par opposition à la française. L'idée d'une naissance des États-Unis sans violence a été défendue par de nombreux historiens d'outre-Atlantique, mais combattue aussi par d'autres qui ont parlé de "guerre civile " au milieu de la guerre contre l'occupant britannique. Il est vrai qu'il n'y a pas eu de massacre systématique de loyalistes par les patriotes, ni l'inverse. La longueur de la montée de la tension avant la rupture complète avec la Grande-Bretagne, une dizaine d'années de 1764 au début de 1776, a permis des compromis provisoires et accommodements entre partisans d'opinion opposée, et des effacements temporaires de la part des amis du gouvernement anglais, pour revenir ensuite discrètement. De même, le vaste territoire disponible donnait la possibilité de changer de lieu au moins pour un moment, ce qui est moins facile dans un pays de forte densité de population et d'encadrement administratif serré. C'est d'abord dans les ports, qui ne rassemblent qu'une petite minorité du peuplement, que le conflit a été fort et il ne fut pas permanent. Néanmoins, d'abord c'est par la pression administrative et le harcèlement populaire que nombre de tories ont été neutralisés et sont souvent restés passifs. Les destructions de stocks de marchandises britanniques et surtout les spectaculaires goudronnages et emplumages ne sont pas anodins et furent accompagnés de blessures et assassinats. Les biens des loyalistes avérés furent saisis et l'exil concerna une fraction de population proportionnellement plus élevée que ce que représente l'émigration en France après 1789. Certains optèrent pour la lutte armée aux côtés de l'armée anglaise, les évaluations de leur nombre varient entre 20000 et 50000 , ce qui n'est pas insignifiant. Ils se livrèrent à des opérations de guérilla avec brûlements de maisons et exécutions d'adversaires auxquelles répliqua une contre-guérilla avec les mêmes pratiques, sans l'approbation explicite des autorités d'État, mais avec parfois leur complicité.

Par contre, la violence pendant la Révolution française n'est niée par personne, à la fois celle des foules et celle institutionnalisée des tribunaux criminels et surtout des juridictions d'exception, à commencer par le Tribunal révolutionnaire. Sur le nombre des victimes on en est toujours au chiffre approximatif de 40000 évalué en 1955 par D. Greer, le maximum étant atteint lors des deux mois d'application de la Grande Terreur de prairial. S'y ajoutent les morts bien plus nombreux encore, mais sujets à vive controverse, de la Vendée. Il est classique d'attribuer l'essentiel des décimations à la responsabilité des Jacobins et à la période où ils sont particulièrement influents, d'août 1792 à juillet 1794. Mais, selon M. Biard et J.-J. Clère (Biard), la Révolution bourgeoise 
modérée n'est pas tendre avec ses adversaires populaires et démocrates. À cet égard, la répression par exécutions judiciaires et fusillades a commencé dans les villes dès l'été 1789 comme l'indiquent Ch. Le Bozec et É. Saunier à propos de la Normandie (Biard). Les premières compagnies de gardes nationales sont formées contre des «brigands " qui viennent surtout des quartiers et faubourgs peuplés d'artisans et journaliers. S'il est vrai que les journées d'octobre 1789 ont précipité l'adoption de la loi martiale du 21 octobre, la préparation de celle-ci a commencé dès septembre. Sans parler de l'affaire du Champ de Mars de 1791, la loi martiale est utilisée ou au moins justifie les opérations de maintien de l'ordre de 1790-1792 contre les manifestants pour le pain et surtout contre les paysans qui exigent la suppression complète des droits seigneuriaux. Lors de ces déploiements de forces, les gardes nationales urbaines tirent facilement sur les foules. Abrogée sous la période jacobine, cette loi martiale réapparaît sous les thermidoriens avec la loi de grande police du 21 mars 1795. Lors du 12 germinal an III à Paris, la Convention n'a encore à sa disposition que la garde nationale, mais commandée énergiquement par Pichegru, et elle peut procéder à 1600 arrestations ou désarmements de "terroristes». Des troupes étant rassemblées ensuite autour de Paris, au 1er prairial ce sont 40000 hommes sous le général Menou, auxquels s'ajoutent 10000 gardes nationaux des quartiers riches et 1500 muscadins, qui sont employés. 8 000 arrestations ou poursuites judiciaires sont effectuées et en procédure accélérée les commissions militaires décident une quarantaine de peines de mort. Dès décembre 1795, le régime nouveau du Directoire se dote d'un ministère de la police. Quant à la Contre-Révolution, elle n'est pas plus tendre, particulièrement pendant les débuts $d u$ soulèvement vendéen contre les gardes nationaux et les administrateurs révolutionnaires. Il faut compter aussi les blessés et fugitifs massacrés lors des opérations de chouannerie en plusieurs provinces et les assassinats de fonctionnaires et acheteurs de biens nationaux. À l'étranger, les gouvernements monarchiques n'ont pas plus de clémence, que ce soit dans les Provinces-Unies en 1787 à la suite de l'invasion de l'armée prussienne, en Angleterre à partir de la fin 1792 et surtout de 1795. En Irlande, terre semi-coloniale pour les Britanniques, il y eut certes des atrocités commises par les rebelles en 1798, en particulier des vengeances par des catholiques contre des familles protestantes, mais la répression menée par l'armée anglaise fut pire. Selon P. Dupuy (Biard), 30 églises furent brûlées bien que le clergé ait dans son ensemble désapprouvé le soulèvement, d'innombrables maisons et fermes détruites, des exécutions sommaires, des jugements expéditifs de cours martiales fondés parfois sur de faux témoignages prononçant peines de mort et peines de 100 à 500 coups de fouet. Au total, on s'accorde généralement à avancer les chiffres de 3450 déportations et 25 à 30000 morts. Dans les colonies, le racisme spontané aidant, administration et colons font souvent pire comme le montre P. Ragon (Biard) avec l'écrasement de la révolte de Tupac-Amaru.

18 Cependant, la révolution est bien plus que le renversement et le meurtre des anciennes élites dirigeantes. Elle est l'établissement d'une organisation sociopolitique nouvelle, en direction de la démocratie, caractère majeur des trois révolutions de la période. Dans les treize colonies, le droit de vote, quoique censitaire, est assez largement répandu, mais les circonscriptions étaient déséquilibrées, ce qui conduisait à des régimes oligarchiques. Les réformes intérieures menées de 1776 à 1784 abaissent le cens électoral et le cens d'éligibilité, ou font disparaitre ce dernier, et le nombre des sièges aux assemblées est augmenté, ce qui diminue le poids des grandes familles de l'Est. La Révolution française, on le sait, est plus novatrice : très réduite dans l'Ancien 
Régime, l'élection est introduite dans tous les rouages administratifs et même dans le judiciaire et le religieux, et on arrive en 1792 à un suffrage masculin quasi universel dont même le Consulat, en dépit de son autoritarisme, sera obligé de se souvenir, tout en le maîtrisant. Deuxième caractéristique positive de toute révolution: une redistribution de la principale richesse de l'époque, la terre. Aux États-Unis, c'est la confiscation des biens des loyalistes, mais surtout, soupape de sûreté sociale qui contribua à atténuer l'ampleur des tensions internes, l'ouverture des immenses espaces à l'ouest des Appalaches organisée encore sous la Confédération par les lois de 1785 et 1787. En France, bien plus que le partage des communaux, c'est la vente des biens nationaux, problème ancien revisité récemment ${ }^{8}$. Il s'agit de plus de $10 \%$ de la surface agricole utile et dont la majeure partie est cédée de 1790 à $1795 ; 35$ à $40 \%$, donc davantage que ce que l'on estimait auparavant, revenant à la paysannerie. L'Église en ressort gravement affaiblie et la noblesse, en dépit de divers subterfuges, y perd aussi, quoiqu'elle réussisse à préserver l'essentiel de sa fortune.

19 Autre question présentée préférentiellement dans ces livres: le rôle des classes populaires dans le déroulement des révolutions et de leur radicalisation. Dans les treize colonies, dès le début des heurts avec l'Angleterre, en 1765, les ouvriers, dockers, marins, artisans des ports se montrent nettement plus hostiles aux Britanniques que les élites dirigeantes. Comme le remarque E. Marienstras (Monnier), ils sont plus attachés au pays nouveau et ceux des petits fermiers qui se joignent à eux vont l'être encore plus que les négociants et grands planteurs que les affaires et l'éducation lient à la GrandeBretagne. Les premiers plus que les seconds vont avoir à supporter jusqu'à 1775 le poids de plus en plus lourd de l'occupation militaire anglaise avec la perception des taxes, le chômage dû aux blocus divers, le logement des soldats, la concurrence de ces derniers pour le travail et leurs brutalités. C'est sous leur pression croissante, surtout après 1768, que l'idée de rupture va s'imposer, décision à laquelle l'oligarchie ne se rallie complètement que au début de 1776 . On aurait pu également poser le problème de l'explication de la résistance américaine sans aide extérieure marquante jusqu'à 1779 comme fait remarquer Ch. Hermann (Bourdin - Bianchi), avec une armée improvisée et manquant d'argent, face aux Anglais disposant de soldats, d'un commandement et d'une marine composés de professionnels, ainsi que de moyens financiers relativement abondants. Ce rapport de forces défavorable suppose un soutien populaire pour parvenir à en neutraliser les effets les plus défavorables.

20 Évidemment, à propos de l'intervention populaire dans la Révolution française, sansculotterie et paysannerie, à partir du 10 juillet 1789 si ce n'est même avant le soulèvement de la Révolution municipale, et jusqu'au printemps 1794 où apparaît la coupure entre la Convention montagnarde et le peuple des villes et où certaines revendications agraires des petits paysans sur la terre, malgré les lois de ventôse qui ne connaîtront guère d'application, et les réclamations sur les baux fonciers demeurent en suspens. Au printemps 1795, la réaction thermidorienne va repousser définitivement ces réclamations. Sur ce schéma de l'évolution, l'accord entre les auteurs est presque unanime, même s'il y a des nuances entre eux. L'abandon par F. Furet et D. Richet de la césure à l'intérieur de la Révolution symbolisée par le "dérapage ", et partant la reconnaissance d'une continuité entre 1789 et 1794, la multiplication des monographies régionales et locales montrant la réalité de la politisation jusque dans les campagnes $\left({ }^{9}\right)$, la mesure de l'extension impressionnante du réseau des sociétés populaires $\left({ }^{10}\right)$, une meilleure approche de l'ampleur des mouvements paysans avec la publication du livre de A. Ado ( $\left.{ }^{11}\right)$, tout cela oblige à cesser de ne voir dans les Jacobins qu'une minorité 
agissante dirigeant les événements au gré de son idéologie. La Révolution apparaitt bien comme un phénomène de mobilisation de masse qui a commencé avant même la création des clubs. Comme l'écrit Ph. Bourdin (Bourdin), le mouvement populaire hérite et prolonge des formes d'agitation, émeutes et révoltes, d'Ancien Régime, mais tout à la fois il pousse en avant vers les réformes et innovations politiques et sociales la révolution d'assemblée, celle de la bourgeoisie, et dans une dialectique incessante jusqu'à 1794 il se nourrit, se renforce et prend une dimension et une organisation nouvelles en s'emparant des décisions législatives et des institutions administratives du pouvoir légal. A contrario, le Directoire, selon S. Bianchi (Bianchi), réévalué récemment, s'il n'apparaît plus aussi stérile qu'on le croyait autrefois, n'en est pas moins un régime condamné à une instabilité qui le discrédite, parce qu'il s'est resserré sur une base sociale des plus étroites, servant des intérêts de classes qui n'étaient plus ceux du mouvement populaire.

21 La conséquence de l'adhésion des foules au phénomène révolutionnaire est la cristallisation du sentiment national. Celui-ci dans ses débuts ne date ni de 1789 pour la France, ni de 1776 pour les États-Unis, ni de 1791 ou 1793 pour la Pologne. Dans les treize colonies peu à peu, dès avant 1763, s'est créée la conscience d'une identité américaine originale que la disparition du danger français à l'issue de la guerre de Sept Ans a renforcée. R. Calvet (Calvet) rappelle que le "Grand Réveil » calviniste, face à l'Anglicanisme officiellement soutenu par le gouvernement de Londres et réputé corrompu, a contribué à l'auto-représentation des Insurgents comme pionniers vivant dans une nature sauvage dans la pureté et la frugalité et luttant contre un État dépravé et tyrannique, écho de la geste des Hébreux, peuple élu de la Bible auquel les colons tendent à s'assimiler. Le Common sense de Th. Paine (1776), livre phare de la révolution des États-Unis comme l'est Qu'est-ce que le tiers état? de Sieyès pour les Français en 1789, reprend cette vision. La Constitution de 1787, relève J.-O. Boudon (Poussou 1), introduit la liberté de conscience et teinte les institutions d'une religiosité civile inspirée du protestantisme. En France, c'est une nation au contraire essentiellement laïque que crée la Révolution après l'échec de la Constitution civile du clergé. Par l'effort qu'il tente en matière d'enseignement, de création d'institutions culturelles et scientifiques, et par l'orgueil messianique de la Grande Nation vis-à-vis des autres peuples qu'exalte sa politique extérieure à partir des Girondins, l'État joue un rôle fondamental dans l'épanouissement du sentiment national.

Dans les Provinces-Unies, en Italie et en Suisse, la fondation des républiques-sœurs développe un sentiment unitaire reposant sur l'adhésion à une réforme de la société et de l'État conforme aux principes énoncés par la propagande républicaine plus que sur la conscience d'une identité historique et culturelle particulière. Mais la déception vient vite à cause de l'occupation militaire et de ses exigences matérielles et, selon $\mathrm{M}$. Vovelle (Bourdin - Bianchi), de l'arrogance des Français, de la timidité fréquemment des réformes engagées, de l'instabilité des nouveaux dirigeants sous la pression changeante de la France tantôt plutôt jacobine, tantôt modérée, et de leurs divisions entre unitaires et fédéralistes. Alors se manifeste une conscience identitaire, mais en réaction contre le régime, contre les patriotes et leur allié français, contre certaines des nouveautés, surtout celles visant à l'affaiblissement des Églises et à la laïcisation de la vie quotidienne. Le clergé et la noblesse exploitent alors la colère populaire et la transforment en mouvement contre-révolutionnaire organisé. Ainsi, la ContreRévolution a des causes complexes et peut s'appuyer sur le petit peuple en utilisant les 
caractères propres à chaque société, par exemple en Italie une forte emprise d'une religiosité démonstrative bousculée par les républicains. De toute façon, l'explication de tels soulèvements qui ont longtemps étonné ne peut plus aujourd'hui être trouvée dans le refus des acquis de la Révolution ou en invoquant le seul facteur religieux, quoiqu'il soit effectivement important et souvent spectaculaire. Du côté des intellectuels, le rejet de ce qui vient de France, après l'avoir accueilli avec ferveur de 1789 à 1792 ou en 1796, contribue à activer l'intérêt apparu dans la seconde moitié du XVIIIe siècle pour le passé national plus ou moins lointain, en Italie pour l'époque romaine dont les premières fouilles ont confirmé la grandeur, et pour la langue en formation de Virgile à Machiavel, selon Ph. Bourdin (Bourdin). Se constitue ainsi un sentiment de nationalité - si ce n'est déjà un nationalisme - grâce à la Révolution française, mais éventuellement contre elle. Dans les départements de la Belgique et de la rive gauche du Rhin, les mêmes pillages et la désinvolture du vainqueur vis-à-vis de l'autonomisme local, comme le montre X. Rousseaux pour la « guerre des paysans » en Flandre en 1797 (Biard), créent également un climat de tension propice au soulèvement contre les autorités révolutionnaires. Quant aux monarchies liguées contre la contamination venue de France et les conquêtes territoriales voulues par Paris, la Révolution, encore par réaction, en échauffe le sentiment national, ce que montre particulièrement le cas de l'Espagne hostile à la France nouvelle dès avant la guerre ouverte en 1793, et qui se veut championne du christianisme dans la tradition de la Reconquista du XVe siècle. Tout imprégnés qu'ils soient par les Lumières, les ministres, Aranda ou Floridablanca, sont imbus d'absolutisme et redoutent l'agitation des foules. La lutte contre l'invasion française en 1794-1795 renforce l'union intérieure autour de la noblesse et du clergé, la fermeture à l'influence d'outre-Pyrénées et le repliement sur la tradition, ce qui marquera le royaume et contribuera à la guerre d'Indépendance de 1808 et au Carlisme au XIXe siècle.

23 La Révolution française fait souffler un vent stimulant pour les peuples dominés non seulement en Europe, tels les Irlandais en 1799 ou les Polonais après le premier partage, mais également dans le monde colonial, en particulier dans l'empire français. La création du premier État résultant de la libération des plus opprimés, les esclaves noirs, avec la République de Haïti en 1804, retient longuement l'attention de la dizaine d'ouvrages. Quatre points majeurs se dégagent des diverses analyses proposées. D'abord accusé d'hypocrisie par divers historiens dans les années 1980 parce qu'il apparaissait timide et prévoyait une longue transition vers la liberté pour les Noirs qu'il jugeait trop immatures pour être immédiatement autonomes, ce qui fleure à l'avance la morale des colonialistes civilisateurs du XIXe siècle, le courant abolitionniste, plus puissant en Angleterre qu'en France, s'est radicalisé à la fin du XVIIIe siècle à mesure que tout l'Ancien Régime était remis en cause. À Paris il ne se limite pas à la durée d'existence de la Société des Amis des Noirs fondée en 1788 et qui disparaît à la fin de 1791. Non seulement les Girondins avant la réunion de la Convention et au début de cette dernière l'ont porté, mais il a touché aussi nombre de Montagnards, sinon on ne comprendrait pas l'adoption du décret d'abolition du 4 février 1794. Mais en face d'eux les abolitionnistes ont trouvé un groupe de colons installé dans la capitale autour de Moreau de Saint Méry dès 1788 et qui, arguant du danger non imaginaire comme le souligne B. Grunberg (AHM) de sécession des îles antillaises, sait s'organiser pour influencer la Constitutante. Sentant le danger que représente la Déclaration des droits de l'homme, ils créent le 20 août 1789 le club Massiac, se rapprochent des députés contre-révolutionnaires, et obtiennent le vote des 
décrets des 13 mai et 24 septembre 1791 qui laissent le soin de légiférer sur les libres de couleurs et les esclaves aux assemblées coloniales. Le club est fermé tardivement, le 9 mars 1794, et ses adhérents théoriquement poursuivis, ce qui ne les empêche pas de reparaître et s'activer dès 1795. Leurs démarches font que l'esclavagisme est à nouveau défendu dans les débats des Conseils à partir de 1797 et que la Constitution de l'an VIII prévoit des lois spéciales pour les colonies, ce qui prépare le retour à l'esclavage en 1802.

Pourtant quelles que soient les décisions de Paris, dans ce domaine comme ailleurs, ce qui est décisif est l'action sur le terrain qui précède les actes législatifs. Certes, des circonstances temporaires ou locales brouillent de temps en temps la situation, telle à la Guadeloupe en 1789-1791 chez les Blancs la rivalité qui vient de l'Ancien Régime pour la possession du monopole d'entrepôt entre Basse-Terre qui se veut patriote et Pointeà-Pitre royaliste. Mais l'important est, du côté des colons, la prise de pouvoir de fait par les assemblées coloniales en 1789, du côté des Noirs la révolte des mulâtres de SaintDomingue en se réclamant de la Révolution en septembre 1790, ce qui pose le problème $\mathrm{du}$ statut des hommes de couleur libres, puis le soulèvement des esclaves de l'île en août 1791 et l'abolition par Sonthonax de l'esclavage en août 1793, ralliant ainsi la masse des Noirs à la République contre les colons et leurs alliés britanniques et espagnols. Comment enfin en est-on arrivé à l'indépendance de Haïti? M. Dorigny (Dorigny) voit agir trois causes principales : le refus par les autorités françaises d'une réforme agraire partageant les plantations, et l'instauration du travail forcé malgré la liberté qui ont détaché les Noirs de la République, ensuite la volonté des élites noires apparues dans les combats qui se sont construit un État dont elles veulent se réserver les bénéfices, enfin le revirement de la politique du Consulat qui envoie une armée de reconquête aux îles en 1801.

Si le sort des Antilles est en relation étroite avec l'évolution de la politique intérieure de la France, comme en témoigne la décision d'abolition de l'esclavage, unique et prise dans la période jacobine, par contre l'écho de la Révolution dans les colonies d'Amérique latine est faible et ne consiste avant les guerres d'indépendance des années 1810 qu'en quelques petits complots. Plutôt que d'absence d'influence de la Révolution dans cette partie du monde, il parait plus exact, comme le suggère S. Bianchi (Bianchi), de parler d'écho différé, car les colons espagnols et portugais avaient comme ceux de Saint Domingue des raisons notables de se soulever contre la métropole et ne s'étaient pas privé de se rebeller avant 1789. Quant aux Indiens, leur sort ne s'améliorant nullement à la fin du XVIIIe siècle, leur colère contre les propriétaires blancs et l'administration royale ne peut pas s'éteindre. On peut, semble-t-il, distinguer trois freins qui ont retardé l'éclatement d'un conflit grave : la profonde imprégnation d'un catholicisme actif et mal disposé vis-à-vis des idéaux nouveaux, une méfiance justifiée contre l'idée révolutionnaire par peur des Indiens et des esclaves que le pouvoir métropolitain aide à maîtriser, le relâchement de la tutelle métropolitaine avec le blocus britannique de 1795 à 1802 et à partir de 1808 qui apaise les conflits avec le gouvernement royal, et qui, contradictoirement, dissout les liens avec la péninsule Ibérique.

IV - Lectures de la Révolution française

26 En dépit de la renommée flatteuse de la Révolution française entretenue par la tradition républicaine au XIXe siècle et encore au XXe siècle, son originalité et l'ampleur des nouveautés qu'elle a apportées ont été remises en cause par un livre 
récent ${ }^{12}$ qui vient lui-même après d'autres. Comme l'écrit J.-P. Poussou (Poussou 1), si la question posée par A. Jourdan est légitime, la réponse donnée, négative, ne parait pas convaincante. Dans le cortège des troubles de la «révolution occidentale ", il y a bien une exception française qui vaut à notre pays une influence particulière en Europe et dans le monde colonial. Son poids à l'époque, aussi bien sur les plans économique, démographique, culturel que politique, fait que ce qui s'y passe intéresse de gré ou de force ses voisins et l'Amérique. Du reste, G. Poumarède (Poussou 2) ne manque pas de souligner que les monarchies européennes ont vu sans déplaisir les premiers troubles français de 1787-1790 qui affaiblissent l'État de Louis XVI et, croyaient-ils, même le prestige intellectuel de Paris. Nulle part ailleurs également, on est allé aussi loin dans la réorganisation de la société et des structures administratives et politiques, tout en proclamant plus nettement qu'aux États-Unis des principes auxquels elle donnait un caractère universel. Enfin, par opposition à la plupart des puissances européennes qui en restaient à des "révolutions d'en haut ", la Révolution française a provoqué le développement d'un mouvement démocratique et égalitariste populaire et pas seulement intellectuel. Dès la fin de 1789 , loin de demeurer un texte de référence lointaine et sans application pratique, la Déclaration des droits de l'homme est devenue un instrument d'action, car, tant en ville dans les conflits du travail qu'à la campagne pour obtenir l'abolition complète des charges seigneuriales, nombre de révoltés s'en sont emparés. En 1793-1794, si se produit bien une dure répression dirigée contre les contre-révolutionnaires et contre tous les opposants, a été mis en place un régime fait d'institutions sociales, culturelles et politiques qui, quoique d'existence brève, a été ensuite une référence mobilisatrice dans la mémoire pour les forces révolutionnaires dans les deux siècles suivants, parce que largement en avance par rapport à ce qui a suivi pendant longtemps, sans qu'il s'agisse déjà de socialisme, mais de lutte contre les injustices. À plus forte raison, même le régime de compromis bourgeois de 1791 a été, bien qu'il ne fut pas recopié fidèlement, un modèle pour les peuples étrangers, en particulier immédiatement les Polonais.

Dès lors que l'on pose un tel constat, on est obligé d'aborder une question complémentaire : les réformes irréversibles imposées par la Révolution française ontelles permis un développement économique et social favorable, c'est-à-dire ont-elles favorisé l'expansion du système capitaliste en libérant l'appareil de production et d'échanges des anciennes contraintes d'origine féodale existant encore dans l'Ancien Régime? En d'autres termes - c'est l'un des grands débats du Bicentenaire - peut-on toujours qualifier la Révolution de « révolution bourgeoise »? J.-P. Poussou (Poussou 1) et R. Calvet (Calvet) analysent systématiquement le problème, tandis que Ph. Bourdin (Bourdin) et S. Bianchi (Bianchi) donnent quelques indications. Aujourd'hui la vision catastrophiste de la Révolution, qui avait connu un regain dans les années 1980-1990, apparaît assez dépassée, sans que la célébration ravie soit devenue la règle pour autant. Sur le plan économique, il est nécessaire de distinguer en premier lieu les effets à court terme qui, en réalité, sont liés davantage à la guerre continuelle de 1792 à 1815 contre des coalitions redoutables. Du côté des dégâts, il y a la ruine du commerce colonial et $\mathrm{du}$ trafic des grands ports qui en vivaient, le marasme de la partie de la proto-industrie qui était tournée vers l'exportation outre-mer, l'inflation galopante de 1791 à 1796 qui tend à appauvrir les salariés et entrave les affaires après les avoir activées. Du côté des effets positifs, on trouve l'expansion du commerce continental avec les conquêtes territoriales et le blocus maritime, l'appel du marché pour la métallurgie et la draperie à destination militaire, et, chapitre pour lequel les données sont encore insuffisantes, 
l'incitation à l'expansion agricole causée par le transfert de propriété dû à la vente des biens nationaux et à l'intensification du marché foncier ordinaire stimulé un moment par l'abondance monétaire. En effet, on ne peut plus suivre toujours la thèse venue de la physiocratie et reprise par G. Lefebvre suivant laquelle la petite propriété est rétrograde techniquement et la grande progressiste. En outre, certaines conséquences de la Révolution n'ont de portée qu'à long terme et ne se manifestent pas au lendemain de 1794 ou 1799 ou même de 1815, mais au fil des décennies. Dans un cadre général qui est évidemment très différent de celui du XXIe siècle, une série de nouveautés et d'institutions créées ou modifiées par la Révolution sont favorables à l'essor de l'esprit d'entreprise et, avec lui, à l'activité et à la modernisation technique. Ainsi l'établissement de la liberté intérieure - sans préjudice d'un éventuel protectionnisme extérieur - contre les corporations, les monopoles de commerce et de fabrication manufacturière et le contrôle de la circulation, la stabilisation de la monnaie avec le franc germinal de 1803, la garantie de l'épargne avec la limitation de

l'endettement de l'État ; l'adoption d'une fiscalité qui pèse davantage sur l'immobilier que sur le mobilier; l'élargissement du marché par l'homogénéisation de l'espace intérieur; l'incitation au crédit par la création d'une banque centrale d'émission, d'escompte et de prêt à l'État, soutenue par celui-ci, ce qui avait manqué à la monarchie ; la mise en place d'institutions de représentation et régulation des affaires avec les Chambres de commerce, les tribunaux de commerce; la sacralisation de la propriété par la sévérité de la répression de tout ce qui peut y porter atteinte, en particulier le vol.

28 Au bilan économique s'ajoutent les résultats sur le plan social : la laïcisation de l'État et de la vie civile avec la liberté de conscience et de culte, l'état civil, le mariage civil et le divorce qui libèrent en partie les individus des contraintes de la famille patriarcale, sans aller jusqu'à l'égalité réelle des femmes. Moins hardi que la législation de la Convention, le Code Civil n'en va pas moins dans le sens plutôt de l'individualisme. Par contre l'éducation, l'assistance et les hôpitaux sont largement abandonnés à l'initiative privée, ce qui est la condition d'une stabilisation du budget de l'État et par conséquent d'une fiscalité respectueuse des intérêts des affaires. Il est vrai qu'après 1794 la Révolution aussi bien que l'Empire sont durs aux pauvres. Sur le plan politique a été mise en place la citoyenneté, mais après 1794 on va vers le régime des notables, compromis entre la propriété foncière débarrassée de la féodalité et la bourgeoisie, qui d'ailleurs ne dédaigne pas la terre.

Cette énumération des résultats de ce qu'il est finalement bien difficile de ne pas appeler une révolution bourgeoise à participation populaire, fait un assez large accord parmi la majorité des historiens participants aux dix volumes. On voit aussi que les positions d'interprétation de la Révolution française ont sensiblement évolué en une vingtaine d'années. En effet, C. Mazauric (Biard) rappelle que, dans les années antérieures au Bicentenaire, la Révolution avait été inscrite au programme de l'agrégation d'histoire de 1984-1985 sous le libellé «L'Europe à la fin du XVIIIe siècle (Russie, pays scandinaves, Empire Ottoman exclus) 1789-1802 », et avait eu lieu à cette occasion une polémique dont on retrouve des traces, car elle reflétait un climat dominant, dans certains colloques et publications sur 1789 qui ont suivi. D'une part, la Révolution n'apparait plus comme le modèle unique de développement historique vers la démocratie politique et sociale, et ses contradictions sont mieux repérées, à l'inverse peu nombreux sont ceux qui y voient encore une dictature mystificatrice et sanglante qui n'aurait apporté que retard d'évolution et malheur. Est-ce à dire que les trois 
courants d'interprétation qui, selon aussi C. Mazauric (Bourdin - Bianchi), se sont constitués au lendemain de l'événement révolutionnaire lui-même et parcourent les XIXe et XXe siècles, aient totalement disparu?

Très minoritaire apparaît maintenant le courant d'origine légitimiste, ultra conservateur, autrefois de teinture royaliste, qui s'est fixé sur son terrain de prédilection dans les années 1980 : le "génocide » de la Vendée. On en retrouve des éléments dans le chapitre rédigé par A. Gérard (Poussou 2). L'auteur n'en est évidemment plus à la vision idyllique du régime seigneurial de la province selon les Mémoires de la marquise de La Rochejacquelin, et il note lui aussi que les paysans de la province ont d'abord été favorables à la Révolution. Toutefois, selon lui et sans donner les preuves de l'affirmation, la Vendée aurait été non seulement une révolte de grande étendue, mais également un instrument entre les mains des Montagnards dans leur lutte contre les Girondins avant le 2 juin 1793. Ils se seraient abstenus de pousser la Convention à ordonner une répression rapide, de façon à compromettre les Girondins alors dominants, ce qui a facilité l'expansion du soulèvement. Puis, maitres du gouvernement, ils se seraient livrés à la fureur purificatrice qui les caractérisait. Seconde idée originale, les Vendéens ne sont pas tombés dans la barbarie de leurs adversaires : ils libéraient leurs prisonniers quand les Bleus les fusillaient. Quant aux généraux et dirigeants politiques qui ont commandé les ravages des «colonnes infernales » et les noyades de Nantes, A. Gérard dégage Tureau d'une partie de ses responsabilités afin de charger le Comité de salut public et Carrier, émanation des Jacobins qui serait «l'archétype des révolutionnaires professionnels». Il reprend ainsi sans distance critique le discours des thermidoriens à la recherche de boucs-émissaires afin de faire oublier leur propre orientation avant la chute de Robespierre, et de se débarrasser d'une partie des Montagnards devenus encombrants.

Deuxième courant d'interprétation, venu de l'Orléanisme et de Thiers, libéral conservateur, rajeuni par F. Furet et prédominant dans les années 1980 : il demeure actif et on le trouve principalement dans les deux livres dirigés par J.-P. Poussou. Par rapport au Bicentenaire, il a évolué et s'est nuancé de façon lui aussi à tenir compte des recherches nouvelles d'archives. Trois traits principaux paraissent le caractériser: le refus proclamé à propos des origines de la Révolution, mais cela déborde la question, de distinguer causes profondes et causes immédiates, position qui est en réalité un refus d'envisager ces causes profondes et le caractère contradictoire des structures en place. Deuxième affirmation : le primat du politique, mais sans nier la possible action dessus des autres instances du tout social. Le dernier trait est la minoration, sans aller jusqu'à l'élimination, du rôle des interventions des catégories populaires dans le champ des décisions politiques, voire culturelles. Concrètement pour l'avant 1789, la crise économique et sociale de l'Ancien Régime n'est vue par J.-P. Poussou que sous l'angle de la crise conjoncturelle de subsistances et de la dépression industrielle de 1788-1789, la décélération et, pour certains secteurs, la stagnation, apparue vers 1770 à côté de la poursuite de la croissance démographique - ce qui implique un déséquilibre structurel ne sont pas envisagées. Par contre, brièvement évoquée, la théorie des élites demeure à l'honneur, entraînant de minimiser le fossé noblesse - bourgeoisie, ce que fait M. Figeac (Poussou 2) avec prudence, les hobereaux restant indécrottables. Pour la «prérévolution » et le premier semestre de 1789, J.-P. Poussou reprend G. Taylor (1967) : la Révolution «fut essentiellement une révolution politique aux conséquences sociales plutôt qu'une révolution sociale aux conséquences politiques " (Poussou 1). Le règne de Louis XVI est un échec quasi-total parce que l'État n'a pas su ni voulu se réformer pour 
s'adapter aux mutations matérielles et sociales en cours. Le gouvernement n'a jamais choisi clairement entre les diverses pressions et solutions possibles aux difficultés, en particulier financières. Il n'a pas contrôlé l'opinion qui lui devient alors de plus en plus hostile, et les notables et les Parlements ont bloqué les mesures nouvelles. Au centre se trouve naturellement la nullité du monarque. Le caractère traditionnel de cette analyse est tempéré par le découpage chronologique qui en est dessiné : la première période 1787-1788 est celle du déclenchement de la grande crise avec la rébellion des Parlements et la révolte nobiliaire dont le rôle est privilégié, puis les États généraux jusqu'à l'Assemblée nationale, et enfin en juillet 1789 l'entrée en scène du peuple des villes et ensuite des paysans. Le monde politique est donc vu comme peu perméable à l'agitation des foules, mais l'auteur concède que la Grande Peur a bousculé l'Assemblée et l'a contrainte à aller plus loin que ne le voulaient les députés. En face, le roi et la reine sont reconnus comme hostiles au Tiers dès la mi-juin 1789. Malgré tout, selon M. Figeac (Poussou 2), la noblesse a pu encore espérer utiliser en 1790 les nouvelles institutions électives pour conserver une position prééminente. N'est-ce pas reconnaître qu'un compromis de fait s'est établi en août 1789 entre noblesse et bourgeoisie et que la poursuite du mouvement populaire l'a remis en cause, en d'autres termes qu'il y a eu une dynamique de... lutte de classes ? Pour la suite de la Révolution après 1789 , J.-P. Poussou note qu'elle recrute de moins en moins de soutien à mesure qu'on avance dans le temps, ce qui est tout de même faire bon marché des travaux déjà cités sur les variations de la participation électorale et sur l'étendue du réseau des sociétés populaires.

On retrouve le même état d'esprit dans l'analyse de la révolution américaine par $\mathrm{E}$. Broglin (Poussou 2). Écrivant que la révolution d'outre-Atlantique avait «su canaliser et limiter la violence ", il prend à son compte l'équivalent aux États-Unis de la théorie française des élites, la thèse du consensus et de la déférence hiérarchique. Après une description brillante de la croissance économique et démographique au XVIIIe siècle, il est ainsi conduit à négliger complètement les conflits sociaux dans les treize colonies avant 1776 et après, bien qu'il ait mentionné en bibliographie (Poussou 1) certains des travaux américains qui ont étudié ce sujet. De même les esclaves et surtout les Indiens sont-ils absents du chapitre, quoique les ouvrages les étudiant soient indiqués. Du même coup l'aiguisement de la différenciation sociale pendant le XVIIIe siècle, la pauvreté dans les ports, la montée de la fortune des grands marchands, l'aristocratisme chez les grands planteurs du Sud, etc., ne sont pas vus, pas plus que les conflits sur la rédaction des constitutions des États après 1776. Cependant, quelques notations sont révélatrices, au-delà des débats doctrinaux, du poids des déterminations sociales. Ainsi la réaction dans les États « contre le danger d'une absolue prédominance de l'élément démocratique et populaire ", et à propos de la préparation de la constitution de 1787 "la montée d'une réelle angoisse chez beaucoup d'Américains ", mots élégants pour traduire la lutte de classe en ne voulant pas l'énoncer tout de go. Dans cette ligne la Constitution fédérale n'aurait pas à voir avec une réaction conservatrice, ce qui étonne lorsqu'on a lu les textes d'époque sur la « mobcracy ». La Convention de Philadelphie ne se situe-t-elle pas en fait dans la suite d'entreprises précédentes, non évoquées par l'auteur, pour une révision des Articles de Confédération. Telle fut la tentative sur le plan financier du superintendant des finances R. Morris d'établissement d'un système fiscal fédéral en 1781-1783, et celle d'A. Hamilton en même temps de réforme de la constitution de la République, personnages que l'on retrouve parmi les tenants du texte de 1787, sans parler de la sédition militaire dite « conspiration de Newburgh » de 1783, 
événement auquel les mêmes paraissent liés, de même que G. Washington, l'un des pères de la Constitution fédérale.

Le chapitre de P. Gueniffey (Poussou 2) relève de la même inspiration, mais portée à l'extrême et plutôt durcie par rapport à son livre de $2000\left({ }^{13}\right)$, au point de rejoindre la première orientation d'interprétation et de rappeler Joseph de Maistre. On entre dans le mystère insondable dès le début : il s'agit de saisir « la part d'énigme » que comporte le cours de la Révolution, tout en professant classiquement une parfaite objectivité, ne pencher ni vers les royalistes ni vers les «héritiers de la Révolution». La Terreur a commencé avant même 1793 et dès 1789 s'est déchaînée la violence spontanée contre la menace de subversion, attisée par une hantise délirante du complot contrerévolutionnaire et par la passion égalitaire. Dans cette atmosphère, le fantasme trouve une belle carrière. 1793 est le temps du terrorisme instrumentalisé par le besoin d'élimination des leaders révolutionnaires les uns par les autres à cause d'une surenchère permanente afin d'arriver à l'impossible : créer l'homme nouveau. Un tel schéma repose sur quelques postulats pour le moins discutables : une sous-estimation de la crise de l'Ancien Régime ( «jamais la monarchie n'avait été aussi libérale et le poids des privilèges aussi négligeable qu'au temps de Louis XVI») et de l'importance de la Contre-Révolution qui n'est pas qu'imaginaire; s'ajoutent un psychologisme banal, renforcé par une psychanalyse collective rétrospective pour sonder l'inconscient des acteurs du passé, et une dissolution de la réalité sociale où n'existent plus qu'idées et principes. De plus, la méthode de P. Gueniffey surprend toujours: une déontologie singulière fait qu'il ne prend jamais en considération - éventuellement pour les réfuter - les travaux de ceux qui ne suivent pas la même orientation. Ici, il aurait pu sembler utile de consulter certains des livres de M. Vovelle ( $\left.{ }^{14}\right)$, et, entre autres, les recherches sur les représentants du peuple en mission ( $\left.{ }^{15}\right)$, on les cherche en vain dans les notes. Et la lecture des sources faite par l'auteur est toujours littérale, alors qu'on sait le gonflement quasi systématique de la réalité auquel se livre le discours révolutionnaire.

Le troisième courant est encore moins formalisé que les deux précédents. Dénommé par ceux qui s'y opposent comme «jacobin » ou «marxiste », mais plutôt républicain démocrate, il vient de L. Blanc en passant par Jaurès pour arriver à G. Lefebvre et comporte des historiens qui se réclament effectivement du marxisme comme hier A. Soboul, aujourd'hui M. Vovelle ou C. Mazauric, mais également beaucoup d'autres qui refusent toute étiquette. Majoritaire aujourd'hui, influencée par les Annales et E. Labrousse, cette orientation marque une préférence pour les études de terrain fréquemment quantitatives et a investi plus récemment le champ culturel avec $\mathrm{M}$. Vovelle, en s'écartant d'une histoire des idées purement intellectuelle. S'y manifeste une reconnaissance du poids de l'économie et de la démographie et du rôle des choix de classe, même si ceux-ci ne sont pas nécessairement appelés en ces termes. Par là, la différence est sensible avec les deux courants précédents. Pour ces historiens, les " circonstances » également, pour expliquer les comportements révolutionnaires, sont tenues pour nécessaires et non suffisantes, en ce sens qu'elles doivent être replacées dans le cadre de structures sociales et mentales de longue durée.

Parmi ces chercheurs, un groupe se détache qu'on retrouve surtout dans le livre dirigé par R. Monnier. Certains de ces auteurs développent une analyse du discours politique dont les techniques sont empruntées à la linguistique et qui permettent incontestablement d'aller plus loin dans l'appréhension de la pensée des émetteurs que l'analyse littéraire formelle. Leurs sources sont puisées principalement dans les débats 
des assemblées législatives et des clubs, les journaux et pamphlets, les cahiers de doléances ou les délibérations municipales. Ainsi la contribution de S. Wahnich est centrée sur l'utilisation du mot " peuple " à travers les documents sur les mouvements populaires urbains. Le récit politique tient dans une telle démarche une place essentielle, et J. Guilhaumou par exemple se propose de "construire une narration continue de l'action révolutionnaire " autour de plusieurs mots comme "action", « révolte» ou «révolution", tandis que R. Monnier veut, par l'histoire des «notionsconcepts ", "retrouver l'histoire politique à la lumière du symbolique et de l'histoire culturelle ». Au point où aujourd'hui arrive cette méthodologie, on peut se demander si elle n'a pas atteint ses limites d'efficacité. Parvenir à la conclusion suivant laquelle la revendication du mouvement populaire précède largement la décision de l'Assemblée constituée, revient un peu à enfoncer une porte ouverte. Coupée de l'étude de l'environnement matériel, économique, démographique et social par une certaine polarisation sur la technique d'analyse et le type de sources dépouillées, cette démarche verse dans un nominalisme qui renvoie à la querelle des réaux et des nominaux du XIIe siècle. Car ces travaux donnent parfois l'impression que c'est la parole qui mène exclusivement l'évolution historique, et qu'il faut et suffit qu'une chose soit dite pour qu'ensuite elle pénètre dans les consciences des contemporains et que le réel total en soit modifié, ce qui est assez parallèle à la vision ultra-conservatrice - quoique partant d'horizons idéologiques opposés - de la Révolution. Bien que ces auteurs essaient de donner aux « circonstances » une place, leurs analyses déploient un spontanéisme implicite qui non seulement minimise les conditionnements autres, mais aussi tend à réduire les contradictions objectives et les conflits collectifs à des batailles oratoires. D'autre part, pour étudier la Révolution paysanne dont on sait par ses travaux antérieurs qu'elle la connaît particulièrement, F. Gauthier (Monnier) en vient à forger des entités verbales de forme allégorique qui rappellent le jeu du Roman de la Rose version Guillaume de Lorris. "À l'offre de la paysannerie de contrat social, la seigneurie répondit en partie par la négative ». Une telle écriture ne risque-t-elle pas de produire un récit trop linéaire et manichéen? Dans cette voie, on risque de tomber sous le coup des mêmes critiques que celles que formulaient les historiens épistémologues des années 1970, tel Michel de Certeau, vis-à-vis de l'histoire « économique et sociale » contre les catégories quantifiées de l'histoire sociale qu'ils jugeaient sans chair humaine et totalement abstraites.

36 En fait, on voit que cette dizaine d'ouvrages parus en même temps sur un même thème, à dire vrai vaste, n'est pas inutile parce qu'elle peut inciter à ouvrir et rouvrir des discussions sur la démarche historienne en général, comme à propos des chantiers concrets de recherche. Comme le signalait G. Noiriel ${ }^{16} \mathrm{il} \mathrm{y} \mathrm{a} \mathrm{peu,} \mathrm{le} \mathrm{débat} \mathrm{prolongé} \mathrm{est}$ une pratique encore peu répandue dans l'historiographie française. Quelque avis que l'on porte sur telle ou telle contribution, on voit que, tout en étant marquées par des continuités idéologiques et par les clivages - ce qui au fond a le mérite d'exciter à la discussion - celles-ci engendrent, ces interventions rendent compte du renouvellement qui apparaît dans l'historiographie française depuis une dizaine d'années et qui se manifeste également dans la dernière synthèse parue sur la Révolution ${ }^{17}$. Sont pris en considération la contradiction dans l'histoire et les conflits entre camps idéologiques et sociaux et à l'intérieur de chacun de ces camps, dans une recherche de la totalité de la formation sociale, ce qui ne se confond pas avec le totalitarisme comme on tendait à le penser vers 1980 . 


\section{NOTES}

1.Direction J.-P. Poussou, Guide. Révoltes et révolutions en Europe et aux Amériques 1773-1802 (avec J.-O. Boudon, E. Broglin, O. Chaline, M. Figeac, A. Gérard, O. Petré-Grenouilleau, G. Pommarède), Paris, Armand Colin, 2004, 250 pages (sera indiqué Poussou 1) ; dir. J.-P. Poussou, Le bouleversement de l'ordre du monde. Révoltes et Révolutions en Europe et aux Amériques à la fin du XVIIIe siècle (avec les mêmes et Y.-M. Bercé, L. Bély, P. Gueniffey), Paris, SEDIS, 2004, 430 pages (Poussou 2) ; dir. Ph. BouRdin, J.-L. CHAPPEY, Révoltes et révolutions en Europe et aux Amériques 1773-1802, (J.-P. Bertaud, St Clay, J. Delinière, P. Dupuy, B. Gainot, R. Hocquellet, H. Leuwers), Paris, SEDES, 2004, 396 pages (Bourdin) ; dir. S. BIANCHI, Ph. BouRDIN, Révoltes et révolutions de 1773 à 1802. Europe, Russie, Amériques (F. Antoine, M. Belissa, J. Bernet, B. Bodinier, Y. Bosc, J. Bourguet-Rouveyre, B. Ciotti, F. Gauthier, M. Gilli, Ch. Hermann, C. Mazauric, M. Vovelle, N. Wulf), Nantes, édit. du Temps, 2004, 382 pages (Bourdin - Bianchi) ; dir. R. MONNIER, Révoltes et révolutions en Europe (Russie comprise) et aux Amériques de 1773 à 1802 (M. Belissa, W. Berelowitch, Y. Bosc, F. Gauthier, J. Guilhaumou, E. Marienstras, F. Marius-Hatchi, J. Rosendaal, S. Wanich), Paris, Ellipses, 2004, 352 pages (Monnier) ; S. BIANCHI, Des révoltes aux révolutions. Europe, Russie, Amérique 1770-1802, Rennes, Presses de l'Université de Rennes, 2004, 488 pages (Bianchi) ; M. DORIGNY, Révoltes et révolutions en Europe et aux Amériques 1773-1802, Paris, Belin, 2004, 174 pages (Dorigny) ; R. CALVET, Révoltes et révolutions en Europe et aux Amériques 1773-1802, Paris, Armand Colin, 2004, 250 pages (Calvet). Il convient d'ajouter les actes du colloque annuel de l'Association des Historiens Modernistes des Universités en octobre 2004, Révoltes et révolutions en Amérique et en Europe 1773-1802 (Y.-M. Bercé, P. Dupuy, A. Duprat, M. Belissa, Ch. Peyrard, Ph. Bourdin, B. Grunberg, A. Berelowich), Paris, Presses de l'Université de Paris-Sorbonne, 2005, 150 pages (A.H.M.) ; les actes de la journée d'études Université-IUFM de Rouen, janvier 2005, dir. M. BIARD, Des révoltes au temps de la Révolution française (C. Mazauric, G. Lemarchand, Ch. Le Bozec, E. Saunier, J.-J. Clère, P. Ragon, F. Régent, X. Rousseau, P. Dupuy, E. Wauters, B. Bodinier, A. Celeri) à paraître 2005 Cahiers d'Histoire - Revue d'histoire critique (Biard). En outre, il faut signaler trois ouvrages à but plus strictement pédagogique : M. BELISSA, Révoltes et révolutions en Europe (Russie comprise) et aux Amériques de 1773 à 1802. Approches de la question, Paris, Hachette-Education, 2004, 192 pages (Recueil d'articles anciens et conseils) ; dir. A. JOLLET, Révoltes et révoltions en Europe (Russie incluse) et aux Amériques de 1773 à 1802, en dissertations corrigées, Paris, Ellipses, 2005 (avec 12 collaborateurs), 266 pages ; Pierre BENOIST, Révoltes et Révolutions. Documents, Atlande Neuilly 2005, 320 pages.

2.Bulletin de la Société d'Histoire Moderne et Contemporaine, 1999, 3-4.

3.François CROUZET, La grande inflation. La monnaie en France de Louis XVI à Napoléon, Paris, 1993.

4.Marie-Denise DEMELAS, Nadine VIVIER (dir.), Les propriétés collectives face aux attaques libérales 1750-1914. Europe occidentale et Amérique latine, Rennes, 2003.

5.Jean NiCOLAS, La rébellion française. Mouvements populaires et conscience sociale 1661-1789, Paris, 2002.

6.Timothy TACKETT, Par la volonté du peuple : comment les députés de 1789 sont devenus révolutionnaires, Paris, 1997. 
7.Georges GUSDORF, Les révolutions de France et d'Amérique, la violence et la sagesse, Paris, 1988.

8.Bernard BODINIER, Éric TEYSSIER, L'événement le plus important de la Révolution. La vente des biens nationaux, Paris, 2000.

9.Exemple récent avec Serge BIANCHI, La Révolution et la première République au village. Pouvoirs, votes et politisation dans les campagnes d'Ile de France, Paris, 2003.

10.Jean BOUTIER, Philippe BOUTRY, Serge BONIN (dir.), Les sociétés populaires, fasc. 6 de Atlas de la Révolution française, Paris, 1992.

11.Anatoli ADO, Paysans en Révolution. Terre, pouvoir et jacquerie 1789-1794, traduction, Paris, 1996.

12.Annie Jourdan, La Révolution, une exception française ?, Paris, 2004.

13.Patrice GUENIFFEY, La politique de la Terreur. Essai sur la violence révolutionnaire 1789-1794, Paris, 2000.

14.En particulier M. Vovelle, La mentalité révolutionnaire, Paris, 1986 ; du même La découverte de la politique, Paris 1993. C'est-à-dire des travaux qui ouvrent des pistes qui ne sont pas sans rapport avec la préoccupation de P. Gueniffey.

15.M. BIARD, Missionnaires de la République. Les représentants du peuple en mission 1793-1795, Paris, 2002, livre auquel il paraît difficile de ne pas recourir lorsqu'on étudie la Terreur. 16.Gérard NoIRIEL, Sur la «crise » de l'histoire, Paris, 1996.

17. Michel BIARD, Pascal DuPUY, La Révolution française. Dynamiques, influences, débats 1787-1804, Paris, Armand Colin, 2004, 346 pages.

\section{AUTEUR}

GUY LEMARCHAND

Université de Rouen 